\title{
A Numerical Approach for Solving Quadratic Integral Equations of Urysohn's Type using Radial Basis Function \\ Zakieh Avazzadeh*
}

Faculty of Science, Islamic Azad University, Mashhad Branch, Mashhad, Iran

\begin{abstract}
In this paper, the numerical method to obtain the approximate solution of quadratic integral equations of Urysohn's type is presented. This class of equations is often discussed in the different way focusing on the properties of involving functions in the integral equations. The use of radial basis functions for solving the Fredholm integral equations was offered by Makroglou [1] and we develop the method for solving the quadratic integral equations. In this study, the radial basis functions method with the collocation scheme to obtain the numerical solution of Fredholm and Volterra quadratic integral equations of Urysohn's type is described. This technique plays an important role to reduce a quadratic integral equation to a system of equations. Some illustrative examples are demonstrated which confirm the efficiency, validity and applicability of the presented technique.
\end{abstract}

Keywords: Fredholm and Volterra quadratic integral equations; Urysohn's type; Radial basis functions; Collocation method; Quadrature integration rules

\section{Introduction}

Quadratic integral equations provide an important tool for modeling the numerous problems in engineering and science. These equations appear in the modeling of radiative transfer, kinetic theory of gases, traffic theory, neutron transport and in many other phenomena [2-7]. So, it is clear that solving this class of integral equations can be used to describe many events in the real world. Recently, many different types of research have been focusing on the effective properties of quadratic integral equations such as existence, uniqueness, monotonic solutions and positive solutions of this class of equations [8-13]. There are a few numerical and analytical methods to estimate the solution of the quadratic integral equations such as Picard and Adomian decomposition method (ADM) [14], and some other methods [15].

In this study, the radial basis functions method with the collocation scheme for solving quadratic integral equations of Urysohn's type is described. The use of radial basis functions for solving the Fredholm integral equation was offered by Makroglou [1] and Alipanah and Dehghan [16] facilitated this method with the quadrature integration technique. Also, this method is compared with the method via orthogonal polynomials [17]. We utilize the method for solving the quadratic integral equations.

A nonlinear Fredholm quadratic integral equation of Urysohn's type can be considered as the following general form

$$
u(t)=f(t)+\lambda(t, u(t)) \int_{a}^{b} F(t, s, u(s)) d s, \quad a \leq t, s \leq b,
$$

and Volterra Urysohn quadratic integral equation of the second kind as follows

$$
u(t)=f(t)+\lambda(t, u(t)) \int_{a}^{t} F(t, s, u(s)) d s, \quad a \leq t, s \leq b,
$$

where $u(t)$ is an unknown function, the function $f(t), F(t, s, u(s))$ and $\lambda(t, s)$ are given. Though the different choices of the parameters lead to various problems, the method can afford to approximate the solution of them.

The presented paper is organized in the following way. In Basic definitions section, we review some basic definitions relevant to the radial basis functions and quadrature integration rules which were applied in solving the process. In Description of method section, we describe the method of solving quadratic integral equations by using radial basis functions in details. Some illustrative examples are presented in Numerical examples section. Numerical results confirm the efficiency and high accuracy of the method. Finally, Conclusion Section concludes this paper with the brief summary and more discussion of the numerical results.

\section{Basic Definitions}

In this section, we review some required tools and definitions. Firstly, we introduce radial basis functions as the effective tools for approximation of the given functions. These basis functions approximate the continuous function with exponential rate of convergency $[18,19]$. Also, we remind the quadrature formulae for numerical integration.

\section{Radial basis functions}

Definition (Interpolation by translates of a single function [20]): Given a set of distinct data points $\left\{x_{j}\right\}_{j=1}^{n}$ and corresponding data scalar values $\left\{f_{j}\right\}_{j=1}^{n}$, the basic interpolant is given by

$$
s(x)=\sum_{j=1}^{n} \lambda_{j} \psi\left(x-x_{i}\right),
$$

when the interpolation conditions are imposed as follows:

$$
f\left(x_{i}\right)=s\left(x_{i}\right)=\sum_{j=1}^{n} \lambda_{j} \psi\left(x_{i}-x_{j}\right), \quad i=1,2, \ldots, n .
$$

The unknown coefficient $\lambda_{j}$ is determined by solving the following linear system

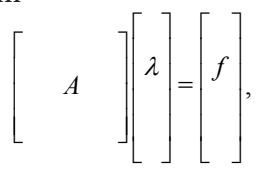

*Corresponding author: Zakieh Avazzadeh, Faculty of Science, Islamic Azad University, Mashhad Branch, Mashhad, Iran, E-mail: z.avazzadeh@yahoo.com

Received June 02, 2012; Accepted July 02, 2012; Published July 05, 2012

Citation: Avazzadeh Z (2012) A Numerical Approach for Solving Quadratic Integral Equations of Urysohn's Type using Radial Basis Functions. J Appl Computat Math 1:116. doi:10.4172/2168-9679.1000116

Copyright: (C) 2012 Avazzadeh Z. This is an open-access article distributed unde the terms of the Creative Commons Attribution License, which permits unrestricted use, distribution, and reproduction in any medium, provided the original author and source are credited. 
where the entries of $A$ are given by

$$
a_{j k}=\psi\left(x_{j}-x_{k}\right)
$$

The interpolant of $f(x)$ is unique if and only if the matrix $A$ is nonsingular.

Radial basis functions (RBF) are the class of single functions whose translates can be useful in multivariate interpolation. RBF methodology was introduced by Hardy [21] and became popular for solving interpolation problems [18,22-29]. The radial basis functions are involved with Euclidean norm as follows:

$$
s(x)=\sum_{j=1}^{n} \lambda_{j} \varphi\left(\mathrm{P} x-x_{i} \mathrm{P}\right),
$$

corresponding to Eq.(3) where $\varphi(r), r>0$. Micchelli [30] gave sufficient conditions for $\varphi(r)$ in Eq.(7) to guarantee that $A$ matrix in Eq.(5) is unconditionally nonsingular. Therefore, the RBF interpolant is uniquely solvable. Some common infinitely smooth examples of the $\varphi(r), r>0$, that lead to a uniquely solvable method are the following forms:

\begin{tabular}{|l|l|}
\hline Gaussian(GA) & $e^{-(\varepsilon r)^{2}}$ \\
\hline Multi Quadric(MQ) & $\left(1+(\varepsilon r)^{2}\right)^{\frac{\alpha}{2}} \quad(\alpha \neq 0, \alpha \neq 2 \mathrm{~N})$ \\
\hline Inverse Multi Quadric(IMQ) & $\left(1+(\varepsilon r)^{2}\right)^{-\frac{1}{2}}$ \\
\hline Inverse Quadric(IQ) & $\left(1+(\varepsilon r)^{2}\right)^{-1}$. \\
\hline
\end{tabular}

Parameter $\varepsilon$ is a parameter for controlling the shape of functions which effects on the rate of convergency. The interested readers can see $[18,20]$ and the references therein. Also, there are some piecewise smooth RBF such as $r^{3}$ (Cubic) and $r^{2} \log r$ (thin plate spline).

It has been discussed about sufficient conditions for $\varphi(r)$ to guarantee nonsingularity of the $A$ matrix $[18,20]$. These conditions show that the larger class of functions could be considered.

\section{Legendre-Gauss-Lobatto (nodes and weights)}

Let $L_{M+1}(\xi)$ be the Legendre polynomial of order $M+1$ on $[-1,1]$ . Then the Legendre-Gauss-Lobatto nodes are

$$
-1<\xi_{0}<\xi_{1}<\ldots<\xi_{M}<1,
$$

where $\xi_{0}=-1, \quad \xi_{M}=1$ and $\xi_{i}, i=1,2, \ldots, M-1$, are the zeros of $L_{M}^{\prime}(\xi)$. No explicit formulas are known for the points $\xi_{i}$, and so they are computed numerically using subroutines. Also, we approximate the integral of $f$ on $[-1,1]$ as :

$$
\int_{-1}^{1} f(\xi) d \xi ; \sum_{i=0}^{M} w_{i} f\left(\xi_{i}\right)
$$

where $\xi_{i}$ denotes Legendre-Guass-Lobatto nodes in Eq.(8) and the weights $w_{i}$ given in [25]

$$
w_{i}=\frac{2}{M(M+1)} \frac{1}{\left[P_{M}\left(\xi_{i}\right)\right]^{2}}, \quad i=0,1, \cdots, M .
$$

Obviously, for arbitrary interval $[a, b]$, we have

$$
\int_{a}^{b} f(\xi) d \xi ; \frac{b-a}{2} \sum_{i=0}^{M} w_{i} f\left(\zeta_{i}\right),
$$

where $\zeta_{i}=\frac{b-a}{2} \xi_{i}+\frac{b+a}{2}$. It is well known that the integration in Eq.(9) is exact whenever $f(\xi)$ is a polynomial of degree $\leq 2 M+1$.

\section{Description of Method}

In 1992, Makroglou [1] proposed the use of radial basis functions for solving the Fredholm integral equation. Alipanah and Dehghan [16] facilitated this method with the quadrature integration technique. In this section, we develop the method for solving the quadratic integral equations.

\section{Fredholm integral equation}

Consider the following Urysohn Fredholm quadratic integral equation

$$
u(t)=f(t)+\lambda(t, u(t)) \int_{a}^{b} F(t, s, u(s)) d s, \quad a \leq t, s \leq b
$$

which is mentioned in Eq.(1). Let $\varphi(x)$ be a radial basis function and we approximate $u(t)$ with the following interpolant

$$
u(t) ; \sum_{j=0}^{n} c_{j} \varphi\left(\mathrm{P} t-t_{j} \mathrm{P}\right)=C^{T} \Psi(t) .
$$

where $C^{T}=\left[c_{0}, c_{1}, \ldots, c_{n}\right]$ and $\Psi^{T}=\left[\varphi\left(\mathrm{P} t-t_{0} \mathrm{P}\right), \varphi\left(\mathrm{P} t-t_{1} \mathrm{P}\right), \ldots, \varphi\left(\mathrm{P} t-t_{n} \mathrm{P}\right)\right]$. Now, by replacing Eq.(13) in Eq.(12) we obtain

$$
C^{T} \Psi(t)=f(t)+\lambda\left(t, C^{T} \Psi(t)\right) \int_{a}^{b} F\left(t, s, C^{T} \Psi(s)\right) d s .
$$

To obtain $c_{j}, j=0,1, \ldots, n$, as unknowns in above equation, we collocate the points $t_{i}, i=0,1, \ldots, n$, such as follows

$$
C^{T} \Psi\left(t_{i}\right)=f\left(t_{i}\right)+\lambda\left(t_{i}, C^{T} \Psi\left(t_{i}\right)\right) \int_{a}^{b} F\left(t_{i}, C^{T} \Psi(s)\right) d s .
$$

By applying quadrature integration formula described in Eq.(11) we can rewrite Eq.(15) in the following form

$$
\begin{aligned}
& C^{T} \Psi\left(t_{i}\right)=f\left(t_{i}\right)+\lambda\left(t_{i}, C^{T} \Psi\left(t_{i}\right)\right) \sum_{j=0}^{N} w_{j} F\left(t_{i}, \tau_{j}, C^{T} \Psi\left(\tau_{j}\right)\right), \\
& i, j=0,1, \ldots, n,
\end{aligned}
$$

where $w_{j}$ and $\tau_{j}, j=0,1, \ldots, n$, are weights and nodes of Legendre-Gauss-Lobatto integration rule. This is a nonlinear system of equation that can be solved by the Newton's iteration method to obtain the unknown vector $C^{T}$.

\section{Volterra integral equation}

Consider the following quadratic integral equation of Volterra type

$$
u(t)=f(t)+\lambda(t, u(t)) \int_{a}^{t} F(t, s, u(s)) d s, a \leq t, s \leq b,
$$

which is mentioned in Eq.(2). Similarly, we substitute Eq.(13) in Eq.(17) and collocate the points $t_{i}, i=0,1, \ldots, n$. So we have

$$
C^{T} \Psi\left(t_{i}\right)=f\left(t_{i}\right)+\lambda\left(t_{i}, C^{T} \Psi\left(t_{i}\right)\right) \int_{a}^{t_{i}} F\left(t_{i}, s, C^{T} \Psi(s)\right) d s .
$$

In above equation, we let $s=g(v)=\frac{t_{i}-a}{2} v+\frac{t_{i}+a}{2}$. It reduces Eq.(18) to the following equation 


$$
\begin{aligned}
& C^{T} \Psi\left(t_{i}\right)=f\left(t_{i}\right)+\frac{t_{i}-a}{2} \lambda\left(t_{i}, C^{T} \Psi\left(t_{i}\right)\right) \int_{-1}^{1} F\left(t_{i}, t_{i} g(v),\right. \\
& \left.C^{T} \Psi\left(t_{i} g(v)\right)\right) d v .
\end{aligned}
$$

Now, by applying Legendre-Gauss-Lobatto integration formula demonstrated in Eq.(9), we approximate the integral of Eq.(19) as follows

$$
\begin{aligned}
& C^{T} \Psi\left(t_{i}\right)=f\left(t_{i}\right)+\frac{t_{i}-a}{2} \lambda\left(t_{i}, C^{T} \Psi\left(t_{i}\right)\right) \sum_{j=0}^{N} w_{j} F\left(t_{i}, g\left(\tau_{j}\right),\right. \\
& \left.C^{T} \Psi\left(g\left(\tau_{j}\right)\right)\right),
\end{aligned}
$$

where $w_{j}$ and $\tau_{j}, j=0,1, \ldots, n$, are weights and nodes of the integration rule. Again, we have a nonlinear system of equations that can be solved by the Newton's iteration method to obtain the unknown vector $C^{T}$.

\section{Numerical Examples}

In this section, some examples are considered. The obtained numerical results confirm the validity and efficiency of the proposed method. The associated computations with the examples were performed using MAPLE 13 with 128 digits precision on a Personal Computer. We note that however 64 digits are considered in computations, we demonstrate only 3 digits for the illustrative examples.

Example 1. As the first example consider the following Volterra quadratic integral equation of Urysohn's type [6]

$$
u(t)=t^{2}+u(t) \int_{0}^{t} \frac{(t+s) u(s)}{1+u^{2}(s)} d s
$$

where the exact solution is not given and we construct the approximate solution in the space $\mathrm{C}[0,1]$. According to Eq.(2)

$$
F(t, s, u(s))=\frac{(t+s) u(s)}{1+u^{2}(s)}, \quad \lambda(t, u(t))=u(t), \quad f(t)=t^{2},
$$

and the approximate solution of the integral equation is based on radial basis functions. The approximate solution is demonstrated in Figure 1. Since the exact solution is not given, we proceed the process of investigating of error with the following formula :

$$
r m s=\sqrt{\frac{\sum_{i=1}^{m} \delta\left(t_{i}\right)}{m}},
$$

where
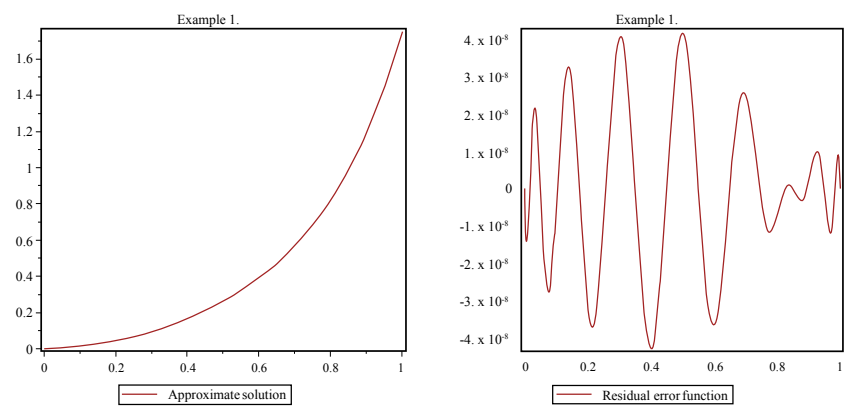

Figure 1: The approximate solution and residual error function for $N=15$. See Example 1.
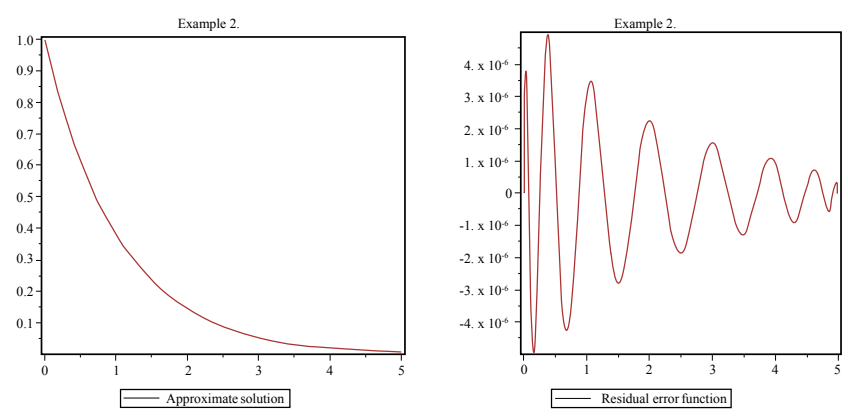

Figure 2: The approximate solution and residual error function for $N=15$ and $T=5$. See Example 2 .

\begin{tabular}{|l|c|c|c|}
\hline & $N=5$ & $N=10$ & $N=15$ \\
\hline Gaussian (GA) & $7.858 \times 10^{-4}$ & $4.718 \times 10^{-6}$ & $2.206 \times 10^{-8}$ \\
\hline Multi Quadratic (MQ) & $7.883 \times 10^{-4}$ & $4.715 \times 10^{-6}$ & $2.216 \times 10^{-8}$ \\
\hline Inverse Quadratic(IQ) & $7.119 \times 10^{-4}$ & $4.715 \times 10^{-6}$ & $2.218 \times 10^{-8}$ \\
\hline
\end{tabular}

Table 1: The rms values for different $N$. The obtained results by using different radial basis functions are similar.

\begin{tabular}{|l|c|c|c|}
\hline & $N=5$ & $N=10$ & $N=15$ \\
\hline $\mathrm{T}=1$ & $5.426 \times 10^{-6}$ & $1.161 \times 10^{-8}$ & $5.172 \times 10^{-12}$ \\
\hline $\mathrm{T}=2$ & $1.112 \times 10^{-4}$ & $1.239 \times 10^{-6}$ & $2.085 \times 10^{-9}$ \\
\hline $\mathrm{T}=5$ & $2.091 \times 10^{-3}$ & $3.504 \times 10^{-5}$ & $1.844 \times 10^{-6}$ \\
\hline
\end{tabular}

Table 2: The $r m s$ values for different $N$ when $T=1,2$ and 5. See Example 2 .

$$
\delta\left(t_{i}\right)=u\left(t_{i}\right)-f\left(t_{i}\right)-\lambda\left(t_{i}, u\left(t_{i}\right)\right) \int F\left(t_{i}, s, u(s)\right) p d s, \quad i=1, \ldots, m,
$$

where $t_{i} \in[a, b]$ can be selected uniformly or randomly and $m$ is a large integer number. The error values for $N=10,15$ and 20 for $m=400$ are illustrated in Table 1 . We solve the problem with multi quadratic (MQ), inverse quadratic (IQ) and Gaussian (GA) radial basis function. The further investigations will be described in the conclusion section.

Example 2. Consider the following Volterra quadratic integral equation of Urysohn's type $[9,31]$

$$
u(t)=e^{-t}+u(t) \int_{0}^{t} \frac{t^{2} \ln (1+s|u(s)|)}{2 e^{t+s}} d s,
$$

where the exact solution is not given and we construct the approximate solution in the space $\mathrm{C}[0, T]$. According to Eq.(2) :

$$
F(t, s, u(s))=\frac{t^{2} \ln (1+s|u(t)|)}{2 e^{t+s}}, \quad \lambda(t, u(t))=u(t), \quad f(t)=e^{-t},
$$

and the approximate solution of the integral equation is based on radial basis functions. The approximate solution is demonstrated in Figure 2. Since the exact solution is not given, we investigate the error values with the rms criterion according to Eq.(21). The error values for $N=5,10$ and 15 for $T=1,2$ and 5 are illustrated in Table 2. The further investigations will be described in the conclusion section.

Example 3. Consider the following Fredholm quadratic integral equation of Urysohn's type [12] 

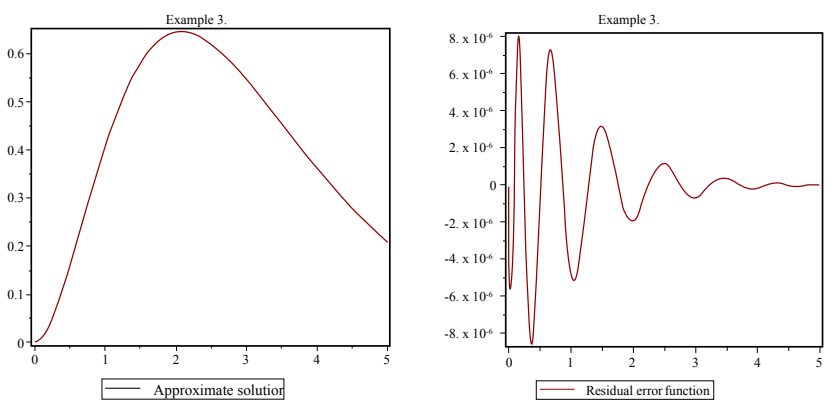

Figure 3: The approximate solution and residual error function for $N=15$ and $T=5$. See Example 3 .

\begin{tabular}{|l|c|c|c|}
\hline & $N=5$ & $N=10$ & $N=15$ \\
\hline $\mathrm{T}=1$ & $1.336 \times 10^{-5}$ & $5.403 \times 10^{-10}$ & $3.062 \times 10^{-13}$ \\
\hline $\mathrm{T}=2$ & $1.797 \times 10^{-4}$ & $7.218 \times 10^{-7}$ & $9.935 \times 10^{-12}$ \\
\hline $\mathrm{T}=5$ & $9.609 \times 10^{-3}$ & $8.583 \times 10^{-5}$ & $8.608 \times 10^{-8}$ \\
\hline
\end{tabular}

Table 3: The rms values for different $N$ when $T=1,2$ and 5 . See Example 3 .

\begin{tabular}{|c|c|c|c|}
\hline Example & $N=5$ & $N=10$ & $N=15$ \\
\hline 4 & $8.280 \times 10^{-5}$ & $9.750 \times 10^{-12}$ & $6.274 \times 10^{-16}$ \\
\hline 5 & $7.342 \times 10^{-6}$ & $5.516 \times 10^{-13}$ & $8.678 \times 10^{-21}$ \\
\hline 6 & $5.763 \times 10^{-4}$ & $1.340 \times 10^{-8}$ & $2.832 \times 10^{-17}$ \\
\hline 7 & $6.651 \times 10^{-6}$ & $4.332 \times 10^{-9}$ & $3.879 \times 10^{-12}$ \\
\hline
\end{tabular}

Table 4: The rms values for different $N$.

$$
u(t)=t^{2} e^{-t}+\frac{t u(t)}{1+t^{2}} \int_{0}^{T} \arctan \left(\frac{t u^{2}(s)}{1+s^{2}}\right) d s,
$$

where the exact solution is not given and we construct the approximate solution in the space $\mathrm{C}[0, T]$ with $0<s, t<T$. According to Eq.(1)

$$
F(t, s, u(s))=\arctan \left(\frac{t u^{2}(s)}{1+s^{2}}\right), \quad \lambda(t, u(t))=\frac{t u(t)}{1+t^{2}}, \quad f(t)=t^{2} e^{-t},
$$

and the approximate solution of the integral equation is based on radial basis functions. The obtained solution is demonstrated in Figure 3 . Since the exact solution is not given, we investigate the error values with the rms criterion according to Eq.(21).

The error values for $N=5,10$ and 15 for $m=400$ are illustrated in Table 3. The further investigations will be described in the conclusion section.

Example 4. Consider the following Fredholm quadratic integral equation of Urysohn's type

$$
u(t)=f(t)+\frac{u(t)}{1+\sin ^{2}(t)} \int_{-1}^{1} \sin (s)\left(u^{2}(s)+t s^{2}\right) d s,
$$

where according to Eq.(1)

$$
F(t, s, u(s))=\sin (s)\left(u^{2}(s)+t s^{2}\right), \quad \lambda(t, u(t))=\frac{u(t)}{1+\sin ^{2}(t)},
$$

and $f(t)$ is chosen such that the exact solution in this example is $u(t)=t \sin (t)-t$. Also, we construct the approximate solution in the space $\mathrm{C}[-1,1]$. The error functions are obtained for $N=10$ and 15 and demonstrated in Figure 4. Also, the rms values for different $N$ are reported in Table 4.

Example 5. Consider the Fredholm quadratic integral equation as follows

$$
u(t)=f(t)+\frac{t u(t)}{\sin (t)+e^{t}} \int_{0}^{1} s^{2} \tanh (t) u(s) d s, \quad t \in[0,1],
$$

where the exact solution is $u(t)=e^{t} \sin t$. According to Eq.(1)

$$
F(t, s, u(s))=s^{2} \tanh (t) u(s), \quad \lambda(t, u(t))=\frac{t u(t)}{\sin t+e^{t}}, \quad f(t)=\frac{e^{t} \sin t(2-t \sinh t)}{2\left(\sin t+e^{t}\right) \cosh t} .
$$

We approximate the solution in the space $C[0,1]$. The error function is shown in Figure 5. Also, the rms values for different $N$ are reported in Table 4 .

Example 6. Consider the Fredholm quadratic integral equation as follows

$u(t)=f(t)+\frac{u(t)}{1+e^{t^{2}}} \int_{0}^{3} s \cos (s) u^{2}(s) d s, \quad t, s \in[0,3]$,

$\begin{aligned} & \text { where according to Eq. } \\ & F(t, s, u(s))=s \cos (s) u^{2}\end{aligned}(s), \quad \lambda(t, u(t))=\frac{u(t)}{1+e^{t^{2}}}$,

and $f(t)$ is chosen so that the exact solution is $u(t)=t \sin t$. We approximate the solution with the radial basis functions in the space $\mathrm{C}[0,3]$. The error function is shown in Figure 6. Also, the rms values for different $N$ are reported in Table 4 .
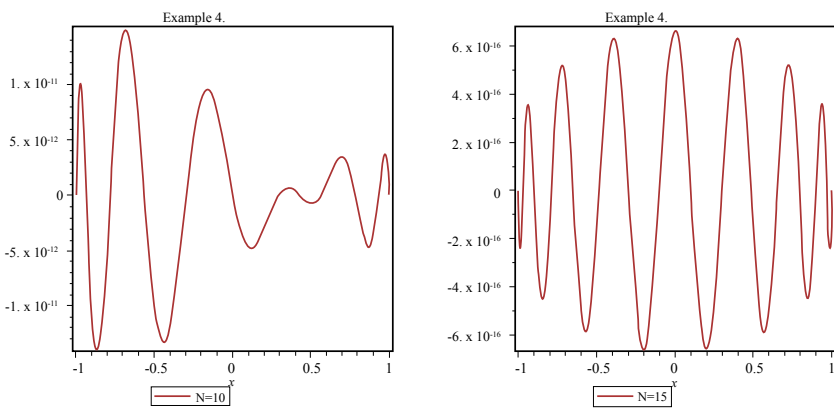

Figure 4: The error functions for $N=10$ and 15. See Example 4.
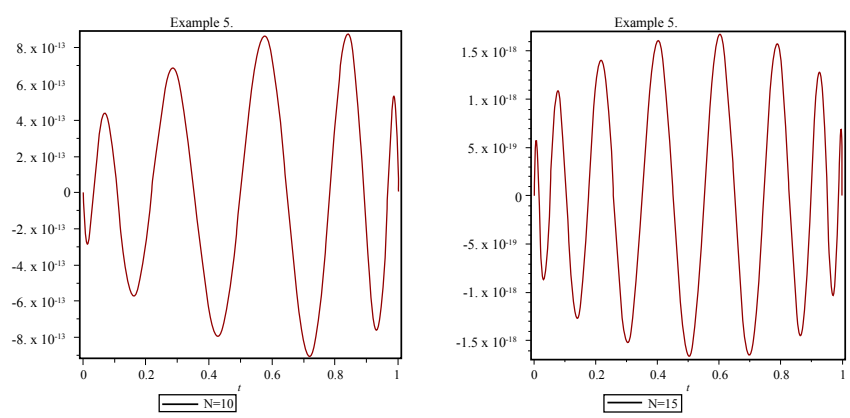

Figure 5: The error functions for $N=10$ and 15 . See Example 5. 

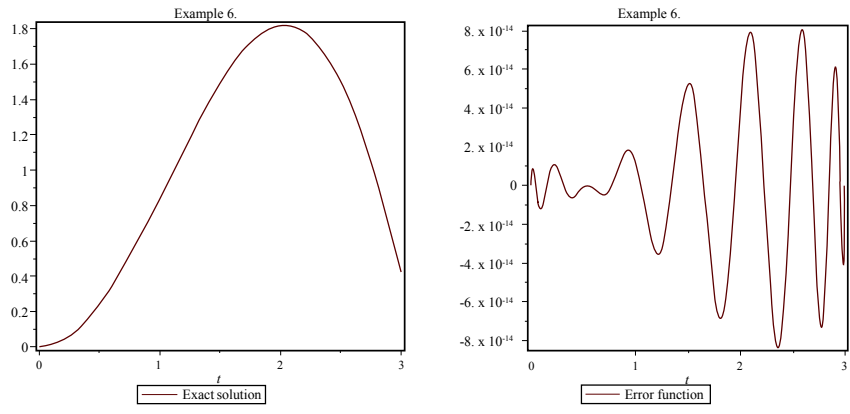

Figure 6: The error function for $N=15$ and the exact solution which is non-monotonic. See Example 6.
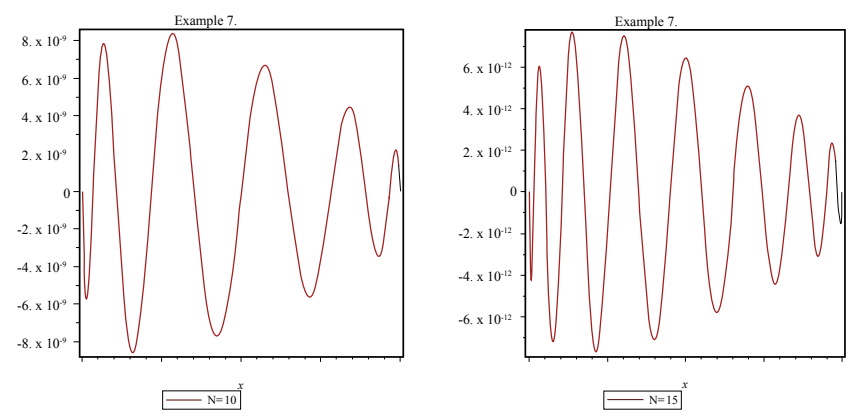

Figure 7: The error functions for $N=10$ and 15 . See Example 7 .

Example 7. Consider the following nonlinear Fredholm quadratic integral equation

$$
u(t)=f(t)+\frac{e^{t} u(t)}{1+e^{t}} \int_{-1}^{1} s u^{2}(s) d s
$$

where according to Eq.(1)

$$
F(t, s, u(s))=s u^{2}(s), \quad \lambda(t, u(t))=\frac{e^{t} u(t)}{1+t^{t}}
$$

and $f(t)$ is chosen such that the exact solution in this example is $u(t)=\frac{1}{3} \sin (\sqrt{t+2})$. Also, we construct the approximate solution in the space C[-1,1]. The error function is obtained for $N=10$ and 15 and demonstrated in Figure 7. Also, the rms values for different $N$ are reported in Table 4.

\section{Conclusion}

The radial basis functions and the collocation method provide the efficient method to solve the general type of linear and nonlinear quadratic integral equations. Moreover, the Urysohn's type of Fredholm and Volterra integral equation can be solved by this method. The proposed method reduces an integral equation to a system of equations. The obtained results showed that this approach can be flexible to solve many different problems effectively.

There are some notable points in numerical results which are considerable. Different radial basis functions can be applicable. Example 1 is solved by multi quadratic (MQ), inverse quadratic (IQ) and Gaussian (GA) radial basis functions that lead to the good results. Although the other examples are solved by different radial basis functions, we only report the obtained results by Gaussian radial basis functions. In addition, number of used basis can be effective on the rate of convergency, see Figures 4,5 and 7. Also, magnitude of domain affects the accuracy. The reported numerical results in Table 2 and 3 is related to $T=1,2$ and 5 . An increase in $T$ causes decrease in the accuracy. Moreover, there are reasonable relationships between rms and absolute error, see Table 4 and Figures 4-7. It will be considerable for cases that exact solution is not given.

Since RBF method uses the norm properties, the presented method can be useful for two dimensional quadratic integral equations. Moreover, the proposed method can be extended to solve the more general types of problem such as quadratic integro-differential equations and systems included quadratic integral equations.

\section{References}

1. Makroglou A (1992) Radial basis functions in the numerical solution of fredholm integral and integro-differential equations. In Advances in Computer Methods for Partial Differential Equations. (VII), New Brunswick, NJ.

2. Argyros IK (1988) On a class of nonlinear integral equations arising in neutron transport. Aequationes Mathematicae 36: 99-111.

3. Busbridge LW (1960) The Mathematics of Radiative Transfer. Cambridge Univ Press, Cambridge, United Kingdom.

4. Case KM, Zweifel PF (1967) Linear Transport Theory. Addison-Wesley, Reading.

5. Chandrasekhar S (1950) Radiative Transfer. London (Oxford Univ Press) 8 393.

6. Hu S, Khavanin M, Zhuang W (1989) Integral equations arising in the kinetic theory of gases. Appl Anal 34: 261-266.

7. Kelly CT (1982) Approximation of solutions of some quadratic integral equations in transport theory. J Integral Equations 4: 221-237.

8. Argyros IK (1985) Quadratic equations and applications to Chandrasekhar's and related equations. Bull Austral Math Soc 32: 275-292.

9. Banas J, Martinon A (2004) Monotonic solutions of a quadratic integral equation of Volterra type. Comput Math Appl 47: 271-279.

10. Banas J, Rodriguez JR, Sadarangani K (2000) On a class of Urysohn-Stieltjes quadratic integral equations and their applications. J Comput Appl Math 113 35-50.

11. Bana J, Lecko M, El-Sayed WG (1998) Existence theorems for some quadratic integral equations. J Math Anal Appl 222: 276-285.

12. El-Sayed WG, Rzepka B (2006) Nondecreasing solutions of a quadratic integral equation of Urysohn type. Comput Math Appl 51: 1065-1074.

13. Pimbley GH (1967) Positive solutions of a quadratic integral equation. 24: 107 127

14. El-Sayed AMA, Hashem HHG (2010) Monotonic positive solution of a nonlinea quadratic functional integral equation. Appl Math Comput 216: 2576-2580.

15. El-Sayed AMA, Saleh MM, Ziada EAA (2008) Numerical and analytic solution for nonlinear quadratic integral equations. Math Sci Res J 12: 183-191.

16. Alipanah A, Dehghan M (2007) Numerical solution of the nonlinear fredholm integral equations by positive definite functions. Appl Math Comput 190: 17541761

17. Avazzadeh Z, Heydari M, Loghmani GB (2011) A comparison between solving two dimensional integral equations by the traditional collocation method and radial basis functions. Appl Math Sci 5: 1145-1152.

18. Buhmann MD (2003) Radial Basis Functions: Theory and Implementations. Cambridge University Press.

19. Madych WR (1992) Miscellaeous error bounds for multiquadratic and related interpolators. Comput Math Appl 24: 121-138.

20. Cheney W, Light W (1999) A Course in Approximation Theory. William Allan New York.

21. Hardy RL (1971) Multiquadratic equation of topology and other irregular surface. J Geophys Res 76: 1905-1915. 
Citation: Avazzadeh Z (2012) A Numerical Approach for Solving Quadratic Integral Equations of Urysohn's Type using Radial Basis Functions. J Appl Computat Math 1:116. doi:10.4172/2168-9679.1000116

Page 6 of 6

22. Buhmann MD (1990) Multivariate interpolation in odd-dimensional euclidean spaces using multiquadrics. Const Approx 6: 21-34

23. Buhmann MD (1990) Multivariate cardinal interpolation with radial basis functions. Constr Approx 6: 225-255.

24. Buhmann MD (1989) "Multivariable Interpolation Using Radial Basis Functions". PhD Dissertation, University of Cambridge, United Kingdom.

25. Jackson IRH (1988) "Radial Basis Function Methods for Multivariable Approximation". PhD Dissertation, University of Cambridge, United Kingdom.

26. Madych WR, Nelson SA (1988) Multivariate interpolation and conditionally positive definite functions. Approx Theory Appl 4: 77-89.
27. Madych WR, Nelson SA (1990) Multivariate interpolation and conditionally positive definite functions. II. Math Comput 54: 211-230.

28. Micchelli CA (1986) Interpolation of scattered data: Distance matrices and conditionally positive definite functions. Constr Approx 2: 11-22.

29. Kythe PK, Schäferkotter MR (2005) Handbook of computational method for integration. Chapman \& Hall/CRC Press.

30. El-sayed AMA, Hashem HHG, Ziada EAA (2010) Picard and Adomian methods for quadratic integral equation. Comp Appl Math 29: 447-463. 